\title{
Zu der Geschichte des colloïdalen Goldes;
}

von

\section{Vanino.}

In den meisten Publikationen, welche sich mit der Herstellung colloïdaler Goldlösungen beschäftigen, wird Faraday als derjenige genannt, der zuerst die Bildung genannter Lösungen beobachtete. Fest steht, daB Faraday zur Herstellung der Lösungen Phosphor verwendete und den Suspensionscharakter derselben erkannte. Aber ohne das Verdienst Faradays schmälern $z u$ wollen, möchte ich doch darauf hinweisen, daß schon die ältere Literatur verschiedene Male auf Vorläufer nach dieser Richtung hinweist. So lesen wir in Junkers Conspectus chemiae 1, 882, daB nach Angaben des Buches sol sine reste der Phosphor das Gold in einen roten Schleim verwandelt und aus Ma cquers chymischem Wörterbuch ersehen wir, daB Zink das Gold purpurrot fällt und der Harn dunkelrot. An anderer Stelle lesen wir auch, $\mathrm{daB}$ das Gold durch starke elektrische Schläge in ein purpurrotes Pulver, welches Glas rot färbt, verwandelt wird. Auch die Schwefelleber wird als Auflösungsmittel des Goldes auf trockenem Wege erwähnt und das Zinnchlorür wird eingehendst als Mittel empfohlen, welches das Gold als purpurfarbenes Pulver (Goldpurpur) fällt und welches der Feuchtigkeit (Lösung) eine Purpurfarbe verleiht (Bergmann). Ohne Zweifel muB auch das trinkbare Gold ${ }^{1}$ ), die Goldtinktur, welche die alten Chemiker hauptsächlich mit Rosmarinöl bereiteten, zu den Vorläufern des colloïdalen Goldes gerechnet werden.

Daß unsere Vorfahren von der Natur dieser Tinkturen die richtige Vorstellung hatten, beweist ferner eine Mitteilung, die wir in dem schon erwähnten Wörterbuche lesen.

1) Über die Darstellung habe ich seinerzeit eingehender in den Berichten der deutschen chemischen Gesellschaft publiziert. Ohne Wasser entstehen bei längerem Stehen grüne Lösungen mit Oberflächenschimmer, mit Wasser farbige Lösungen verschiedener Art. 
576 Vanin 0: Zu der Geschichte des colloidalen Goldes.

"Übrigens", schreibt Macquer, ,sind alle diese Goldtinkturen nichts anderes, als ein überaus feingeteiltes und in einer öligen Flüssigkeit schwimmend gemachtes Gold. Sie sind folglich, eigentlich za reden, keine Tinkturen, und können, wie Herr Baron (Lemery) in seiner Ausgabe von Lemerys Chemie sehr wohl erinnert, auch nur insofern trinkbares Gold genannt werden, insofern man mit diesen Namen keinen anderen Begriff als den verbindet, daB das Gold in einer Flüssigkeit schwimmt und in so feine Teilchen gebracht worden ist, dab es selbst in der Gestalt einer Feuchtigkeit getrunken werden kann."

Interessant in den älteren Literaturangaben sind auch die Urteile über den medizinischen Wert des Goldes. So schreibt Macquer: „Wer auf Goldtinkturen viel bält oder glaubt, dab das Gold vor anderen natürlichen Körpern in Anseben der heilsamen Kräfte etwas voraus habe, so gibt derselbe seine schwache Kenntnis in der Natur zu erkennen und ist von abergläubischen Meinungen nicht weit entfernt."

Plinius ${ }^{1}$ ) war anderer Anschauung. Er berichtet, daB das Gold in vielen Fällen als kräftiges Arzneimittel wirkt.

Gold in den Arzneischatz einzuführen, ist im übrigen schon vielfach versucht worden. Nach $\mathrm{H}$ ag e $r$ tauchten insbesondere im Anfang des 19. Jahrhunderts die alchemistischen Anschauungen über die medizinische Wirkung des Goldes wieder auf. Die vierte Ausgabe des deutschen Arzneibuches hat das letzte Goldpräparat, das Aurum natrium chloratum, ausgeschaltet, aber aus den vorzüglich redigierten Mejerschen Jahrblichern ersehen wir, daB man noch immer Goldpräparate einzuführen versucht. Sogar gegen Trunksucht ist Goldchlorid von Keeley empfohlen worden.

Das Aurum colloïdale bat sich jedoch in der Reihe der modernen Arzneimittel gegenüber dem viel begehrten Argentum colloïdale noch keinen Platz erobert. - Gold in höchst fein verteiltem Zustande ist übrigens schon in der Mitte des vorigen Jahrhunderts von Cretien, einem französischen Arzte, empfohlen worden.

1) Buch 33, Kap. 25. 